\title{
A Simple and Fast Battery Test for Phenotypic Characterization of Mice
}

Mariana S. A. Garcia-Gomes ${ }^{1, \#, ~ *, ~ D e n n i s ~ A . ~ Z a n a t t o 1, ~ \#, ~ P e d r o ~ K . ~ Y a m a m o t o 1 ', ~ D a n i l o ~ W a d t " ~}{ }^{1}$, Ana T. F. B. Antiorio ${ }^{1}$, Jilma Aleman-Laporte', Sandra R. Alexandre-Ribeiro², Guilherme A. Marson³, Cezar Guizzo $^{3}$, Silvia M. G. Massironi ${ }^{1}$, Maria M. Bernardi ${ }^{4}$ and Claudia M. C. Mori ${ }^{1}$

\author{
1Department of Pathology, School of Veterinary Medicine and Animal Science, University of São Paulo \\ (USP), São Paulo, Brazil; '2Department of Immunology, Institute of Biomedical Science, University of \\ São Paulo (USP), São Paulo, Brazil; ${ }^{3}$ Chemistry Department, Institute of Chemistry, University of São \\ Paulo (USP), São Paulo, Brazil; ${ }^{4}$ Graduate Program in Environmental and Experimental Pathology, \\ Paulista University, São Paulo, Brazil \\ *For correspondence: mari-aranha@usp.br \\ \#Contributed equally to this work
}

\begin{abstract}
[Abstract] Despite the great number of test batteries already known to assess the behavior of genetically modified and inbred strains of mice, only a few of them focus on basic neurological parameters. The purpose of the battery test proposed is to settle a specific methodology to characterize the phenotype of neurological disease models in mutant or genetically modified mice. This methodology is simple and efficient in order to analyze several parameters, including general activity, sensory nervous system, sensorimotor system, central nervous system and autonomous nervous system. This can aid the choice of specific additional tests as well as the determination of an interrelationship among phenotypic alterations observed. Although being efficient for a first analysis of a mouse model, interpretation of the results must be carefully made because phenotype manifestation may vary due to many parameters, including mouse strain, environmental and housing condition, animal-experimenter interaction, sample size and tests order. It is important to consider as a critical point if handling procedures are aversive. The results acquired with the analysis of 18 parameters together provide preliminary data to characterize mouse phenotype and helps selecting more specific tests.
\end{abstract}

Keywords: General activity, Sensorimotor system, Central nervous system, Autonomic nervous system, Procedure refinement, Animal models

[Background] Laboratory mice have been used as models for biomedical studies, offering a variety of genetic models such as isogenic strains, spontaneous mutants and genetically modified animals. After the implementation of genetic modification, mice became an essential tool in neuroscience research, allowing researchers to investigate the role of individual genes on development and behavior. Furthermore, mice have been the main mammalian models and genetic organisms due to their $85 \%$ homology when compared to humans (Pritchett-Corning et al., 2015), including similarities with the functionality of the human brain such as anxiety-like behavior and other emotional responses (Van Meer and Raber, 2005). Thus mice became an essential part of efforts in prevention and treatment of 
many diseases, but also to test toxicity and adverse effects of biological and chemical substances and in researching the efficacy and safety of new drugs (McLeod and Hartley, 2017).

Mice behavior can be evaluated in several ways, including direct observation and a wide variety of behavioral tests comprising a single task or multiple tests, using or not automated behavioral response assessment (Wahlsten, 2011). The choice of tests applied in the course of an experiment is directly related to the question to be answered. A battery of tests is proposed to evaluate the basic motor and sensory functions. Some of the tests described in our protocol have been grounded on SHIRPA procedure (Rogers et al., 1997) The open field test (OFT) can be used to evaluate general activity, but also parameters related to sensorimotor, autonomous and sensory nervous systems (Yamamoto et al., 2019). Sensory information provided by the vestibular system is essential in spatial memory process (Manes et al., 2019), sensorimotor activity can be defined as the relationship among sensory perception, movement, emotion and cognition (lliadi et al., 2016). Also, autonomic nervous system is responsible to maintain homeostasis in the organism (McCorry, 2007).

Here, we aim to develop a specific methodology to evaluate behavioral parameters of laboratory mice in order to characterize the phenotype of neurological disease models in mutant or genetically modified mice and to evaluate the effects and toxicity of substances.

\section{Materials and Reagents}

1. Paper towels

2. Custom-made arena built of laminated wood round floor (40 cm diameter) and metallic painted walls $(25 \mathrm{~cm})$

3. Personal protective equipment (e.g., gloves, mask, protective apparel)

4. Wire grid

5. Pen and logbook

6. Laboratory-bred mice

Specific pathogen-free (SPF) mice were obtained from the animal facility of the Department of Immunology, Institute of Biomedical Science, University of São Paulo, Brazil. The animals were housed in individually ventilated cage (Alesco Industry and Commerce, Monte Mor, Brazil) with pine shavings for bedding, under controlled room temperature $(22 \pm 5)^{\circ} \mathrm{C}$ and humidity range from $45 \%$ to $65 \%$. The room was kept on a $12 / 12 \mathrm{~h}$ light/dark cycle (lights on at 07:00 AM) with artificial light. Animals had unrestricted access to filtered and autoclaved water and autoclaved commercial pellets formulated according to the AIN93M rodent diet (Nuvilab, Quimtia, Paraná, Brazil). A total of 26 12-week-old, BALB/cJ mutant cruza pernas ("leg crossing"; abbreviation crup) $(n=13)$ and BALB/cJ $(n=13)$ male mice were used. The crup mutant strain arose from an ENU ( $\mathrm{N}$-ethyl-N-nitrosourea) mutagenesis screen in BALB/cJ genetic background described by Massironi (2006).

7. $5 \%$ ethanol 


\section{Equipment}

1. Anatomic forceps

2. Video camera

3. Fixed iron bar for camera placement above arena center

4. Video tracking system (Noldus Information Technology, model: Ethovision XT software) connected to a personal computer (optional)

\section{Software}

1. Ethovision XT (Noldus, http://www.noldus.com/animal-behavior-research/products/ethovision-xt)

Computer software for automated tracking analysis, or any other software for behavior analysis.

2. Prism 8 (GraphPad Software, http://www.graphpad.com). Computer software for statistical analyses, or any other software for statistical analysis

\section{Procedure}

A. General rules

1. Arena must be placed under indirect light avoiding darkened areas or shadowed zones. The video camera must be placed above and center-aligned with the arena providing an entire coverage within the recording frame.

2. Test must be performed in an isolated room, free from loud noises in order not to disturb animals during the procedure. If a quiet place is not possible using a white noise can be helpful to minimize disturbance from other noises.

3. Animal identification can be written on a paper and placed on the floor, so it is recorded by the video camera during the whole procedure.

4. Keep mice inside their home cages in the test room for $30 \mathrm{~min}$ to acclimation.

5. Before each session, the arena must be cleaned with a $5 \%$ ethanol solution to prevent possible bias caused by odor cues left by the previous mouse.

6. Camera must start recording before animal is placed in the arena center to avoid data loss.

7. Parameters are supposed to be tested in a sequence in order to follow an increasing stress level, so that a first response does not interfere with the following one.

8. All parameters must be evaluated in the same arena, unfamiliar for the animals, aiming to minimize stressful situations for animals. Be sure to take notes of every parameter evaluated. Observational parameters can be analyzed on the video, later. 
B. Test procedure/Data collection

1. After acclimation time, turn on the camera and gently remove mouse from home cage and immediately place it-each mouse, in the same position every time-in the center of the arena, at the test chamber.

2. Once the animal is inside the arena, leave the chamber and record animal activity for $5 \mathrm{~min}$.

3. After recorded activity, return to the chamber in order to start the tests for subsequent parameters. It is not mandatory to record the following part of the procedure.

4. Scores are described in Table 1.

Table 1. Parameters related to the general activity, sensory nervous system, sensorimotor system, central nervous system (CNS) and autonomic nervous system (ANS)

\begin{tabular}{|c|c|c|}
\hline & Parameter & How to measure \\
\hline \multirow{3}{*}{ 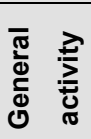 } & Rearing & quantify events (e.g., frequency) \\
\hline & Grooming & quantify events (e.g., frequency, time) \\
\hline & Locomotion & quantify events (e.g., distance, velocity, time) \\
\hline & & Score \\
\hline \multirow{4}{*}{ 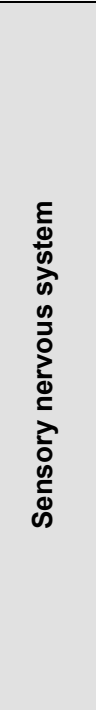 } & Response to touch & $\begin{array}{ll}1 & \text { does not move (absent) } \\
2 & \text { walks with difficulty } \\
3 & \text { walks slowly } \\
4 & \text { walks with agility or runs (baseline) }\end{array}$ \\
\hline & Auricular reflex & $\begin{array}{ll}1 & \text { ears perpendicular to the head and directed forward (absent) } \\
2 & \text { ears rotate outwards and/or back (baseline) } \\
3 & \text { mouse pulls ear back } \\
4 & \text { ears laid flat against the head }\end{array}$ \\
\hline & Corneal reflex & $\begin{array}{ll} & \text { eyes remain open (absent) } \\
2 & \text { eyes half-open } \\
3 & \text { eyes completely closed (baseline) }\end{array}$ \\
\hline & Tail flick & $\begin{array}{ll}1 & \text { no reaction (absent) } \\
2 & \text { moves slowly } \\
3 & \text { moves quickly } \\
4 & \text { run and jump (baseline) }\end{array}$ \\
\hline
\end{tabular}


Please cite this article as: Garcia-Gomes et. al., (2020). A Simple and Fast Battery Test for Phenotypic Characterization of Mice,Bio-protocol 10 (7):

Table 1. Continued

\begin{tabular}{|c|c|c|c|}
\hline \multicolumn{2}{|c|}{ Parameter } & \multicolumn{2}{|c|}{ How to measure } \\
\hline \multirow{4}{*}{ 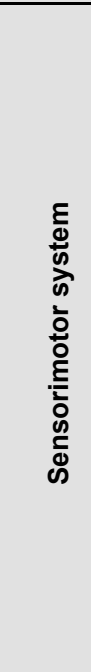 } & Muscle tone & & $\begin{array}{l}\text { absent } \\
\text { present (baseline) }\end{array}$ \\
\hline & $\begin{array}{l}\text { Hindquarter } \\
\text { angulation }\end{array}$ & $\begin{array}{l}1 \\
2 \\
3\end{array}$ & $\begin{array}{l}\text { normal position of the hind-limb and elevated tail (baseline) } \\
\text { increased angle between thoracic back and hip } \\
\text { maximum angle between thoracic back and hip, lowered tail }\end{array}$ \\
\hline & $\begin{array}{l}\text { Surface righting } \\
\text { reflex }\end{array}$ & $\begin{array}{l}2 \\
3 \\
4\end{array}$ & $\begin{array}{l}\text { does not return to prone position (absent) } \\
\text { returns to prone position with difficulty } \\
\text { returns to prone position slowly } \\
\text { returns fast to prone position (baseline) }\end{array}$ \\
\hline & Grasp strength & $\begin{array}{l}1 \\
2 \\
3 \\
4 \\
5\end{array}$ & $\begin{array}{l}\text { does not hold the grid (absent) } \\
\text { hold the grid but quickly release it ( } 2-9 \mathrm{~s}) \\
\text { hold the grid but release it (10-20 s) } \\
\text { hold the grid and then release it }(20-29 \mathrm{~s}) \\
\text { does not release the grid (more than } 30 \mathrm{~s})\end{array}$ \\
\hline \multirow{5}{*}{$\sum_{U}^{\infty}$} & Straub tail & & $\begin{array}{l}\text { absent (baseline) } \\
\text { present }\end{array}$ \\
\hline & Ataxia & & $\begin{array}{l}\text { absent (baseline) } \\
\text { present }\end{array}$ \\
\hline & Tremor & & $\begin{array}{l}\text { absent (baseline) } \\
\text { present }\end{array}$ \\
\hline & Sedation & & $\begin{array}{l}\text { absent (baseline) } \\
\text { present }\end{array}$ \\
\hline & Seizure & & $\begin{array}{l}\text { absent (baseline) } \\
\text { present }\end{array}$ \\
\hline \multirow{2}{*}{$\frac{\infty}{2}$} & Urination & \multicolumn{2}{|r|}{ quantify events (e.g. count urine spots) } \\
\hline & Defecation & \multicolumn{2}{|r|}{ quantify events (e.g. count fecal boli) } \\
\hline
\end{tabular}

Parameters classification was adapted from SHIRPA procedure (Rogers et al., 1997).

\section{Observational parameters (Table 1)}

1. General activity (Video 1) 


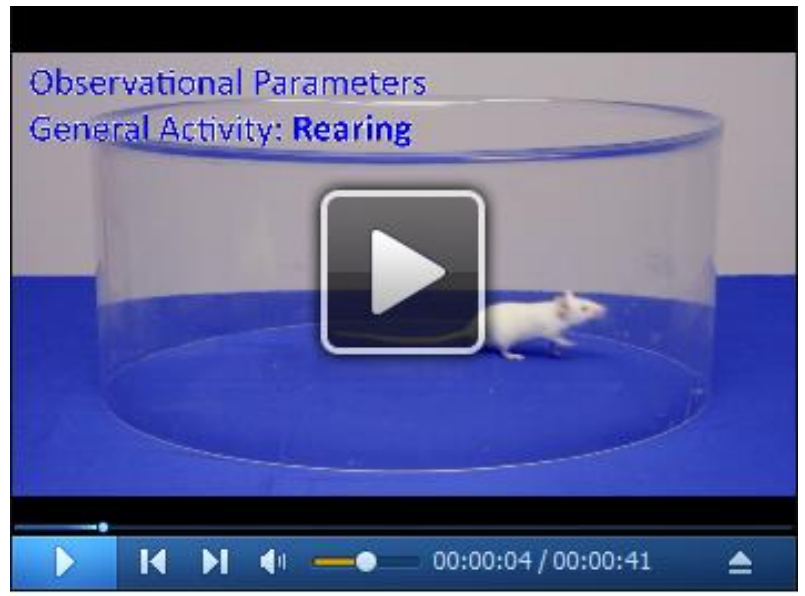

Video 1. Observational Parameters-General activity. Video illustrates general activity parameters: rearing, grooming, and locomotion.

General activity parameters are supposed to be quantified. Locomotion can be analyzed by software (blank arena) or by directly counting sectors covered by animals in an arena having a grid-drawn floor.

a. Rearing. Frequency of standing up events. Animal stands with or without leaning paws on arena walls.

b. Grooming. Frequency and period of self-grooming activity. Animal cleans its face and/or body.

c. Locomotion: Animal walking activity in the arena.

2. Sensorimotor system

a. Muscle tone. Animal presents continuous and passive partial muscle contraction, in order to maintain its body posture (Video 2).

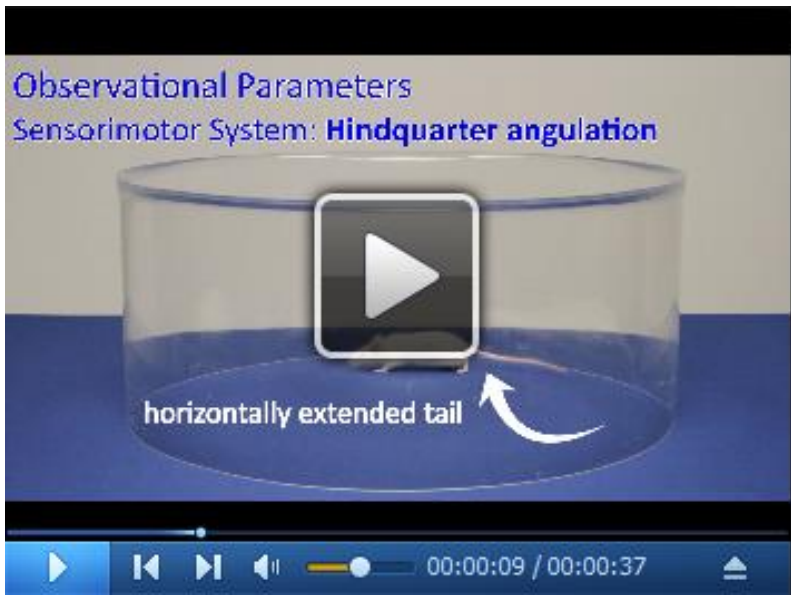

Video 2. Observational Parameters-Sensorimotor system. Video illustrates hindquarter angulation, an observational parameter related to the sensorimotor system. 
b. Hindquarter angulation. Animal presents an increased hindquarter angulation, which can lead to walking difficulties.

3. Central nervous system (Video 3)

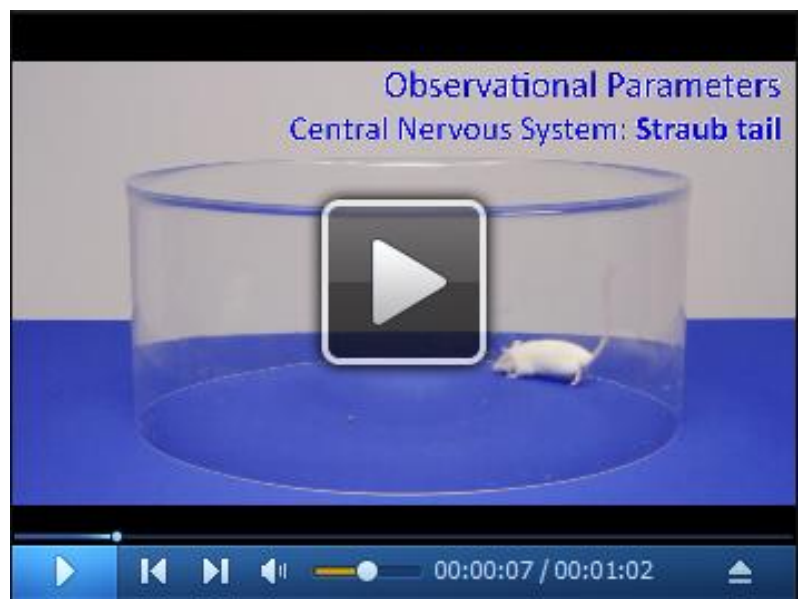

Video 3. Observational Parameters-Central Nervous System. Video illustrates observational parameters related to central nervous system: straub tail, ataxia and tremor.
a. Straub tail. Tail becomes rigid and erect when animal is walking and/or resting
b. Ataxia. Lack of voluntary coordination of muscle movements, gait abnormality.
c. Tremor. Animal presents tremor when walking and/or resting.

4. Autonomic nervous system
a. Urination. Number of urine spots.
b. Defecation. Number of fecal boli.

D. Interventional parameters

1. Sensory nervous system (Video 4)

Response to touch. Gently approach forceps to the animal body, touching it by lateral position for more than $15 \mathrm{~s}$. Observe mouse reaction. 


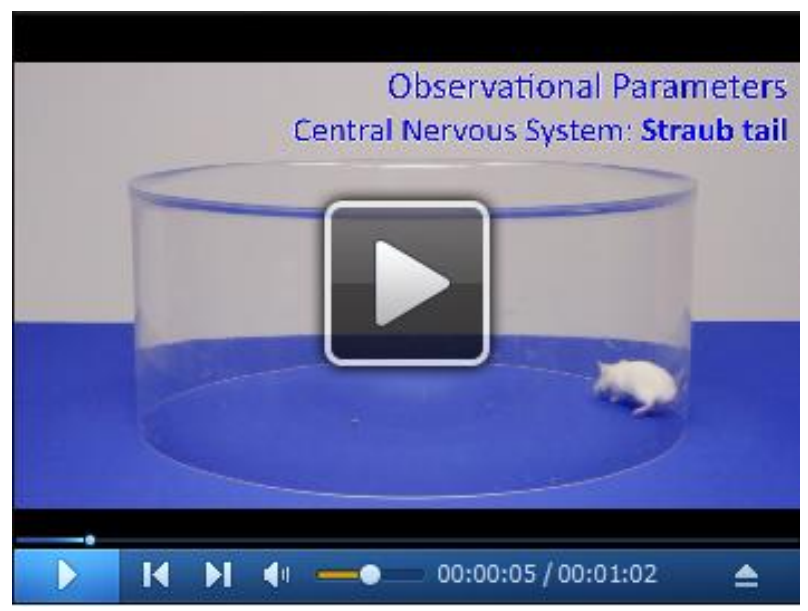

Video 4. Interventional (need the interference of a stimulus [Skinner, 1950]) ParametersSensory Nervous System. Video illustrates interventional parameters related to sensory nervous system: response to touch [score 2-walks slowly and score 4-walks with agility or runs (baseline)]; auricular reflex [score 1 (absent) and score 4-ears rotate outwards and/or back (baseline)]; corneal reflex [score 3-eyes completely closed (baseline)] and tail flick [score 4-run a d jump (baseline).

a. Auricular reflex. Approaching from the side, snap fingers near the ear of the animal and evaluate ears stance.

b. Corneal reflex. Gently approximate the forceps close to the eye of the animal but do not touch it. Be careful to avoid touching whiskers and observe mouse reaction.

c. Tail flick. With forceps tightly press the tip of the tail and observe mouse reaction.

2. Sensorimotor system (Video 5)

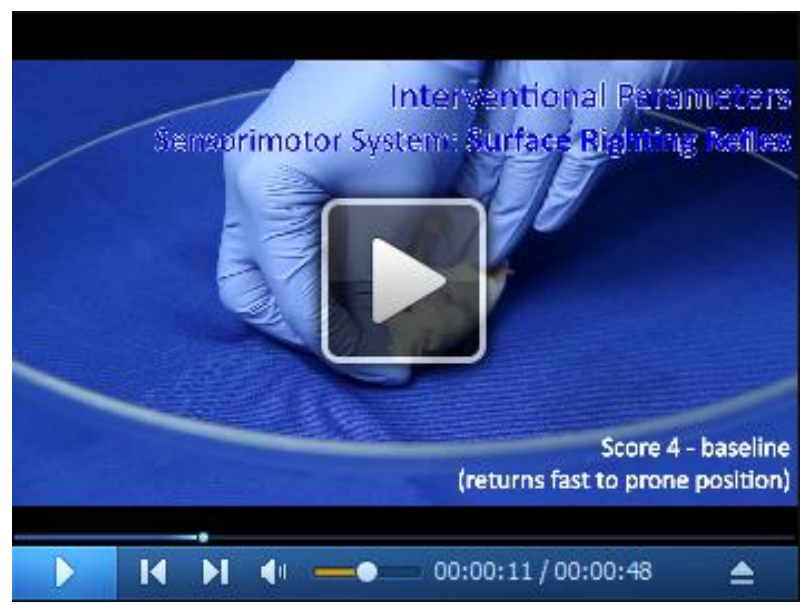

Video 5. Interventional Parameters-Sensorimotor System. Video illustrates interventional parameters related to sensorimotor system: surface righting reflex [score 4-returns fast to prone position (baseline)] and grasp strength [score 1-does not hold the grid (absent) and score 2-holds the grid but quickly release it (2-9 s)]. 
a. Surface righting reflex. Gently turn the animal into its back and place it in supine position. Hold it for $5 \mathrm{~s}$, then analyze latency to return to prone position.

b. Grasp strength. Place animal over the wire grid. Give the animal $5 \mathrm{~s}$ to adjust to the situation, then slowly turn the grid. Observe the animal reaction.

3. Central nervous system

a. Sedation. (If animal is resting, closed eyes) gently touch the animal trunk with the forceps and observe if it moves or open its eyes (Video 6).

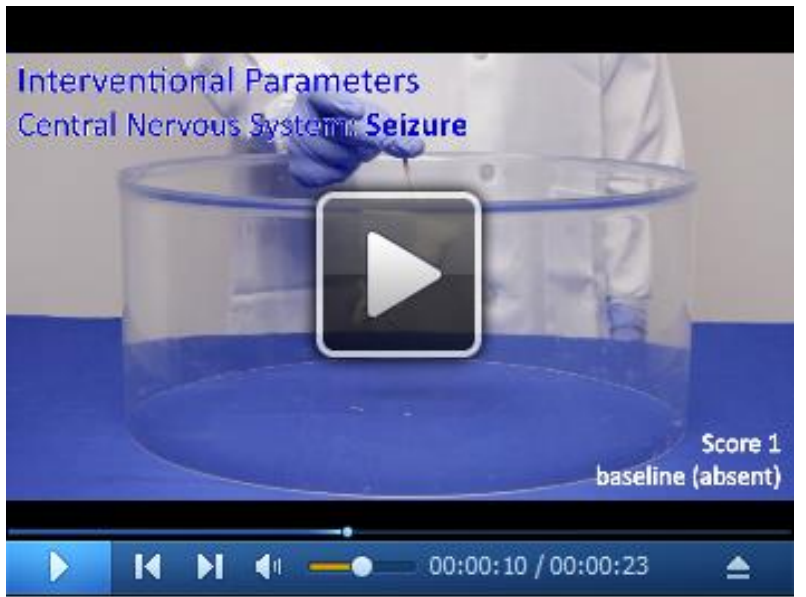

Video 6. Interventional Parameters-Central Nervous System. Video illustrates the procedure to induce seizure, an interventional parameter related to central nervous system: score 1-absent (baseline) was showed.

b. Seizure. Grab mice by tail and gently hang it, spin between fingers, and gently place animal back in the arena; analyze the reaction.

\section{Data analysis}

1. Quantitative-Rearing (frequency), grooming (frequency and/or time), locomotion (distance in $\mathrm{cm}$-using software, e.g., Ethovision ${ }^{\circledR}$-and/or counting square units), urination (number of urine spots), defecation (number of fecal boli).

2. Qualitative-Auricular reflex (score), corneal reflex (score), response to touch (score), tail flick (score), muscle tone (absent/present), hindquarter angulation (score), surface righting reflex (score), grasp strength (score), Straub tail (absent/present), ataxia (absent/present), sedation (absent/present), seizure (absent/present).

3. For statistical analyses, Mann-Whitney (for nonparametric variables, e.g., scores) or Unpaired $t$-test (for parametric variables, e.g., frequency) can be performed using statistical analysis software. 


\section{Representative Results}

In order to illustrate the use of this protocol to characterize a mouse strain with sensorimotor impairment, representative results were exerted from a preliminary study with the recessive mutation crup. BALB/cJ mice were used as control group.

Regarding general activity, the crup mutant showed lower rearing $(\mathrm{t}=4.221, \mathrm{df}=28, P=0.0002)$, grooming frequency $(\mathrm{t}=5.453, \mathrm{df}=24, P<0.0001)$, and decreased distance traveled $(\mathrm{t}=6.124, \mathrm{df}$ $=24, P<0.0001)$ and average speed $(\mathrm{t}=6.109, \mathrm{df}=24, P<0.0001)$ compared with BALB/cJ mice (Figure 1).

A

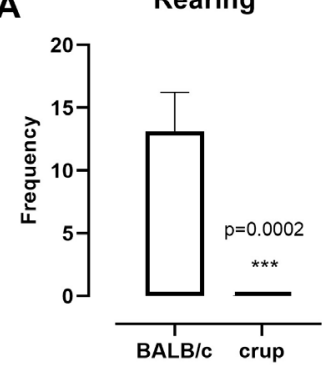

C

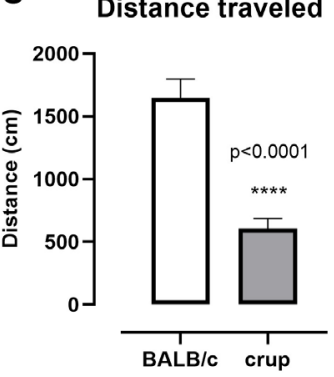

B Grooming

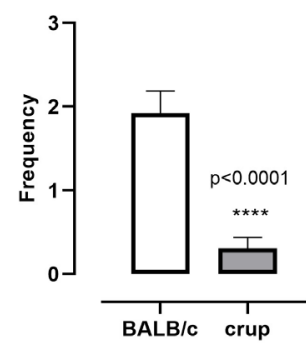

D Average speed

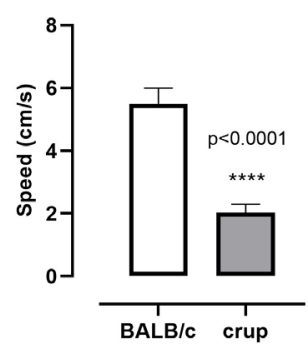

Figure 1. Representative results displaying general activity in the open field in BALB/cJ and crup mutant mice ( $n=13 /$ group). A. Rearing frequency; B. Grooming frequency; C. Distance travelled, locomotion; D. Average speed. Data are presented as the means \pm SEM. Unpaired $t$-test was employed to evaluate strain differences, $P<0.05$.

The crup mutant presented decreased corneal reflex $(P=0.0023, U=30.50)$, compared with BALB/cJ mice. Other parameters involved in the sensory system, such as auricular reflex $(U=$ $62.50)$, response to touch $(U=57.50)$ and tail flick $(U=66.50)$, did not show differences between the strains (Figure 2). 
A Auricular reflex

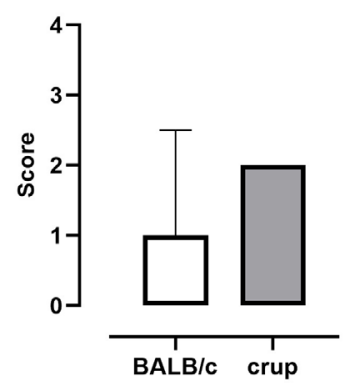

C

Response to touch

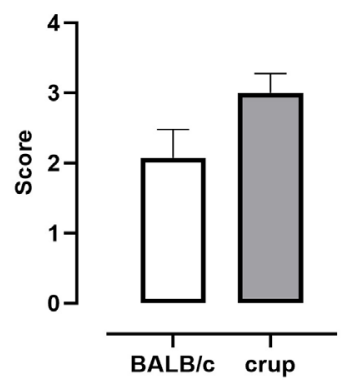

B Corneal reflex

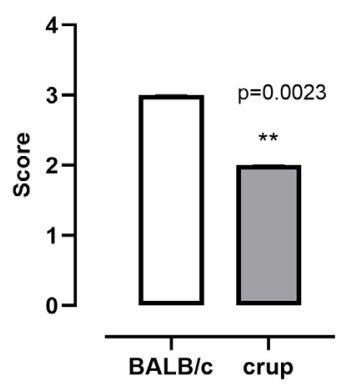

D

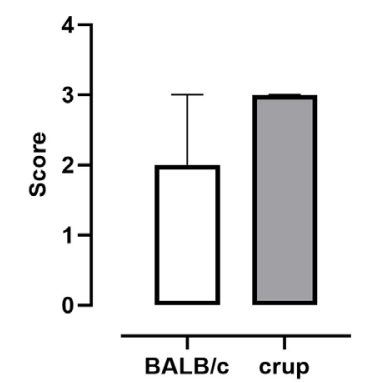

Figure 2. Representative results showing parameters related to the sensory system in BALB/cJ and crup mutant mice ( $n=13 /$ group). A. Auricular reflex; B. Corneal reflex; C. Response to touch and $\mathrm{D}$. tail flick. Data are presented as the median with interquartile range. Mann-Whitney test was employed to evaluate strain differences, $P<0.05$.

Analysis of the sensorimotor system showed reduction in the hindquarter angulation $(P<0.0001, \mathrm{U}$ $=0)$, surface righting reflex $(P<0.0001, U=0)$ and grasp strength $(P<0.0001, U=0)$ in crup mice compared with BALB/cJ controls (Figure 3 ). 
A Hindquarter angulation

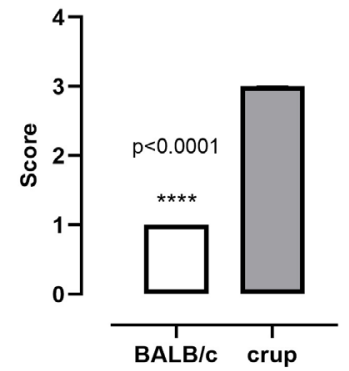

B Surface righting reflex

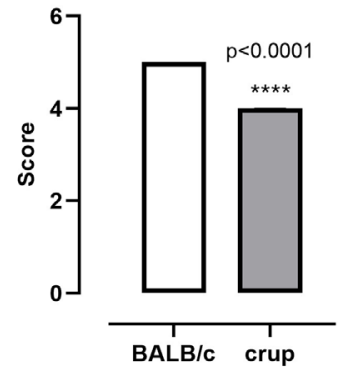

C Grasp strength

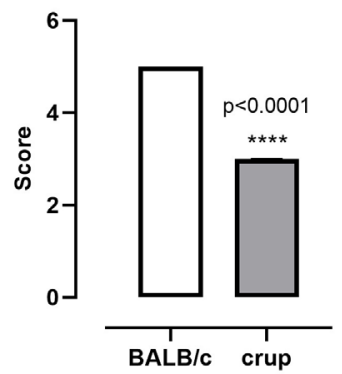

Figure 3. Representative results showing parameters related to the sensorimotor system in BALB/cJ and crup mutant mice ( $n=13$ /group). A. Hindquarter angulation; $B$. Surface righting reflex; $\mathrm{C}$. Grasp strength. Data are presented as the median with interquartile range. Mann-Whitney test was employed to evaluate strain differences, $P<0.05$.

For parameters using scores we used Mann-Whitney test and for the other parameters we used unpaired $t$-test.

Regarding autonomic nervous system parameters, the crup mutant exhibited decreased urination $(\mathrm{t}=2.312, \mathrm{df}=24, P=0.0297)$ and defecation $(\mathrm{t}=6.853$, $\mathrm{df}=24, P<0.0001)$ compared with BALB/cJ (Figure 4).

A Urination

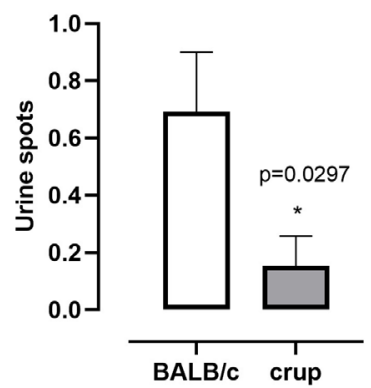

B

Defecation

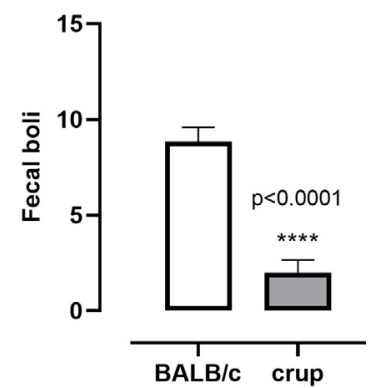

Figure 4. Representative results showing parameters related to the autonomic nervous system in BALB/cJ and crup mutant mice ( $n=13 /$ group). A. Urination; B. Defecation. Data are presented as the means \pm SEM. Unpaired $t$-test was employed to evaluate strain differences, $P<0.05$. 


\section{$\underline{\text { Notes }}$}

1. The arena employed for this test could be the same one used for open field test.

2. Tests should be performed at the same time every day (e.g., in the morning) and by the same person, whose is blind about the animal phenotype. Parameters must be tested with no resting time between the battery. Animals can be tested on different days, under similar conditions, to be compared.

3. This protocol is designed to screen behavioral phenotype of mice with genetic alterations, neurodegenerative diseases function or drug research and development. Tests cover basic parameters to evaluate general neurological characteristics, including sensorimotor and sensory capabilities, as well as autonomic and central nervous system function.

4. Considering the complexity of responses arising from the observation of each parameter, data interpretation must be done carefully, while also considering that phenotype manifestation may vary due to body weight, health status, physical condition, circadian rhythm, genetic background, gender or age; e.g., behavioral changes may worsen with aging, which would present different results if animals are analyzed in different life stages. Multifactorial interference should be taken in account, such as environmental and housing condition, animal-experimenter interaction, sample size and the order tests are conducted (Hånell and Marklund, 2014).

5. General activity can be remotely recorded, which enables automated analysis, but the other parameters require in spot evaluation.

6. Tests must be applied in the open field arena, rather than home cages, for two major reasons: first all animals are supposed to be exposed to the same unfamiliar environment (Whishaw et al., 1999); second, several tests require handling and intervention, which is facilitated in an open field setup.

7. Normal exploratory behavior is measured by calculating horizontal locomotion and vertical activities, which is applied to assess basic motor information that along with other parameters, may provide a more accurate data about mouse phenotype.

8. Sensory nervous system evaluation is an important step to characterize genetically modified or spontaneous mutant mice strains. Thus, the observation of sensory deficits might help to elucidate its development (Hånell and Marklund, 2014). Sensory parameters can be measured by animal reflexes and responses to specific stimuli related to body and proprioceptive senses, that evince evoked brain potentials (Sousa and Almeida, 2006).

9. In this protocol, sensory function is assessed by direct measurement of reflexes, such as response to auditory (auricular reflex), visual (corneal reflex) and somatosensory (response to touch and tail flick) stimuli. Sensorimotor system parameters-muscle tone, hindquarter angulation, surface righting-reflex and grasp strength-can provide information about cerebellar function or asymmetrical brain function. However, grasp strength can also be influenced by body weight (Sousa and Almeida, 2006). 
Detecting sensory or sensorimotor deficits in a preliminary screen might support the choice of specific behavior tests, as animal physical disabilities are considered.

10. In general, more than one task can be selected to test a type of behavior relevant to a hypothesized function of a gene product (Crawley, 1999). An example of our approach is represented in the behavioral analyses of crup mutant mice, which presents remarkable reduction in general activity when compared to BALB/cJ mice. Sensorimotor impairment is indicated by lower scores in surface righting-reflex and grasp strength, and higher scores in hindquarter angulation compared to BALB/cJ. Regarding sensory function, crup showed decreased corneal reflex besides a reduction in urination and defecation, parameters linked to autonomic nervous system. This protocol allows an accurate and comprehensive behavioral analysis suggesting a degenerative process from a preliminary study in the recessive mutant strain crup. The current representative results show behavior genetics analyses that focus on the role of a mutant gene. The appropriate behavior response depends on reflex and sensory capabilities. The initial analysis can suggest indications for other tests that lead to true cognitive deficits (Sousa and Almeida, 2006) and can be expanded for further investigation.

A critical point when applying behavioral test batteries is the interference that the animal suffers if handling procedures are aversive. The testing sequence can attenuate such interference if those are taken following an increasing stress ladder. For example, first general activity is observed in the open field arena for environment habituation followed by observational parameters. Being the most stressful, interventional parameters must be the last to be observed, thus stress from one test will not interfere on the following test response.

This protocol addresses the analysis of 18 parameters together that provides preliminary data to characterize mouse phenotype and helps in choosing more specific tests. Although some of these tasks have already been described separately, here they were presented as a test battery. The following advantages are highlighted: inexpensive; quick and easy-to-perform protocols; combined parameters designed to cover a large number of behaviors displayed by animals; leads to a behavioral fingerprint for a vast range of disabilities.

\section{Acknowledgments}

This protocol is based upon work supported by Fundação de Amparo à Pesquisa do Estado de São Paulo (FAPESP) under grant number 2017/21103-3, and Coordenação de Aperfeiçoamento de Pessoal de Nível Superior - Brasil (CAPES) - Finance Code 001. The authors would like to thank Mr. Ricardo Antiorio for technical assistance within video-record, and Ms. Marina FA Landi for the English revision. This protocol was adapted from Manes et al. (2019) and Yamamoto et al. (2019).

\section{Competing interests}

The authors declare no conflicts of interest or competing interests. 


\section{Ethics}

Experiments on animals were approved by the Animal Care and Use Ethics Committee of the School of Veterinary Medicine and Animal Science, University of São Paulo, Brazil (protocol number 3773120116, FMVZ-USP) and the Institute of Biomedical Science under protocol number 053, page 32, book 3 (2015).

\section{$\underline{\text { References }}$}

1. Crawley, J. N. (1999). Behavioral phenotyping of transgenic and knockout mice: experimental design and evaluation of general health, sensory functions, motor abilities, and specific behavioral tests. Brain Res 835(1): 18-26.

2. Hånell, A. and Marklund, N. (2014). Structured evaluation of rodent behavioral tests used in drug discovery research. Front Behav Neurosci 8: 252.

3. Iliadi, K. G., Gluscencova, O. B. and Boulianne, G. L. (2016). Psychomotor behavior: a practical approach in Drosophila. Front Psychiatry 7: 153.

4. Manes, M., Garcia-Gomes, M. S. A., Sandini, T. M., Zaccarelli-Magalhães, J., Florio, J. C., Alexandre-Ribeiro, S. R., Wadt, D., Bernardi, M. M., Massironi, S. M. G. and Mori, C. M. C. (2019). Behavioral and neurochemical characterization of the mlh mutant mice lacking otoconia. Behav Brain Res 359: 958-966.

5. McCorry, L. K. (2007). Physiology of the autonomic nervous system. Am J Pharm Educ 71(4): 78.

6. McLeod, C. and Hartley, S. (2018). Responsibility and laboratory animal research governance. Sci Technol Human Values 43(4): 723-741.

7. Pritchett-Corning, K., Chou, S., Conour, L. and Elder, B. (2011). Guidebook on mouse and rat colony management.

8. Rogers, D. C., Fisher, E. M., Brown, S. D., Peters, J., Hunter, A. J. and Martin, J. E. (1997). Behavioral and functional analysis of mouse phenotype: SHIRPA, a proposed protocol for comprehensive phenotype assessment. Mamm Genome 8(10): 711-713.

9. Skinner, B. F. (1950). Science and human behavior. vol. 28. doi:10.2307/3707860.

10. Sousa, N., Almeida, O. F. and Wotjak, C. T. (2006). A hitchhiker's guide to behavioral analysis in laboratory rodents. Genes Brain Behav 5 Suppl 2: 5-24.

11. Van Meer, P. and Raber, J. (2005). Mouse behavioural analysis in systems biology. Biochem $J$ 389(Pt 3): 593-610.

12. Wahlsten. D. (2011). Mouse behavioral testing. London, Academic Press.

13. Whishaw, I. Q., Haun, F. and Kolb, B. (1999). Analysis of behavior in laboratory rodents. In: Windhorst U., Johansson H. (Eds.). In: Modern Techniques in Neuroscience Research. Springer, Berlin, Heidelberg. 
14. Yamamoto, P. K., de Souza, T. A., Antiorio, A., Zanatto, D. A., Garcia-Gomes, M. S. A., Alexandre-Ribeiro, S. R., Oliveira, N. S., Menck, C. F. M., Bernardi, M. M., Massironi, S. M. G. and Mori, C. M. C. (2019). Genetic and behavioral characterization of a Kmt2d mouse mutant, a new model for Kabuki Syndrome. Genes Brain Behav 18(8): e12568. 